\title{
Eosinophil count and neutrophil-to-lymphocyte count ratio as biomarkers for predicting early- onset neonatal sepsis
}

\author{
Jang Hoon Lee, MD \\ Department of Pediatrics, Ajou University School of Medicine, Suwon, Korea
}

Despite advances in neonatal intensive care, sepsis remains a major cause of neonatal death and morbidity. Culture-confirmed early-onset neonatal sepsis (EONS), defined as that occurring within 48-72 hours after birth, was determined to have an incidence of 0.4-0.8 per 1,000 live-born term infants in a culture study of developed countries. ${ }^{1)}$ However, the use of systemic antibiotics in infants with culture-negative EONS is reportedly 6-16 times higher than in infants with culture-confirmed EONS.,3)

Many biomarkers such as C-reactive protein (CRP), procalcitonin (PCT), and interleukins have been used to identify infants with sepsis and in clinical decision-making for their mana gement. CRP, a widely used biomarker in the clinical setting, has been extensively studied in research for newborn infants, although it is highly nonspecific as it reportedly increases up to 40-50 mg/L in infants delivered vaginally. ${ }^{4)}$ PCT, a promising biomarker for sepsis in newborn infants, has the advantage of not being affected by maternal fever during labor because of its inability to cross the placenta. ${ }^{5}$ However, few studies have reported the clinical usefulness of PCT for the early diagnosis of EONS.

Eosinopenia, a historical biomarker for infection, has been investigated to differentiate sepsis in non-infectious conditions. Shaaban et al. ${ }^{6}$ reported that an eosinopenia count $<50$ cells/ $\mathrm{mm}^{3}$ showed a sensitivity of $81 \%$, specificity of 65\%, positive predictive value (PPV) of 66\%, and negative predictive value (NPV) of $80 \%$ for predicting sepsis in adults and concluded that eosinopenia is a very sensitive but nonspecific marker of sepsis in the intensive care setting. Wibrow et al. ${ }^{7)}$ reported that eosinophil counts had very little overall predictive ability (area under the receiver operating characteristic curve [AUROC], 0.448; 95\% confidence interval [CI], $0.363-0.533 ; P=0.237$ ) for sepsis, while sensitivity (54\%; 95\% CI, 47\%-61\%) and specificity (56\%; 95\% CI, 49\%-63\%) of eosinopenia $<10 / \mathrm{mm}^{3}$ to predict bloodstream infection in pediatric patients were both low.

The neutrophil-to-lymphocyte count ratio (NLCR), a simple marker of inflammation, is useful for identifying many diseases including sepsis in adults. Dursun et al. ${ }^{8)}$ reported that NLCR had a sensitivity of 75.6\%, specificity of 38.4\%, PPV of 35.6\%, and NPV of $77.8 \%$ to predict sepsis in children. Westerdijk et al. ${ }^{9)}$ reported that the NLCR in adults with sepsis was significantly higher than in those without sepsis (median [interquartile range]:15.3 [10.8-38.2] vs. 9.3 [6.2-14.5], $P<0.001)$ but that the AUROC was significantly higher for CRP $(0.89 ; 95 \%$ CI, 0.87-0.92) and PCT (0.88; 95\% CI, 0.86-0.91) than NLCR (0.66; 95\% CI, 0.62-0.71).

A recent study of the diagnostic value of eosinopenia and NLCR as biomarkers of EONS indicated that eosinopenia of $<140$ cells $/ \mathrm{mm}^{3}$ had a sensitivity of $60.0 \%$, specificity of $90.0 \%$, PPV of 94.7\%, and NPV of 42.9\%, while an NLCR with a 1.245 cutoff value had a sensitivity of 83.3\%, specificity of 93.3\%, PPV of 94.7\%, and NPV of 65.2\%. ${ }^{10)}$ However, this study should be interpreted with caution. First, bacteria were identified only in 20\% (18 of 90) of patients in the EONS group, which differs from the diagnostic criteria for EONS, whereby EONS is defined
Corresponding author: Jang Hoon Lee, M.D. Department of Pediatrics, Ajou University School of Medicine, 164 Worldcup-ro, Yeongtong-gu, Suwon 16499, Korea

Tel: +82-31-219-5167

Fax: +82-31-219-5169

E-mail: neopedlee@ajou.ac.kr

https://orcid.org/0000-0003-4765-9948

Received: 25 March, 2019

Revised: 9 June, 2019

Accepted: 2 July, 2019

See the article "Diagnostic value of eosinopenia and neutrophil to lymphocyte ratio on early onset neonatal sepsis" in Volume 62 on page 217.

Copyright (c) 2019 by The Korean Pediatric Society

This is an open-access article distributed under the terms of the Creative Commons Attribution NonCommercial License (http://creativecommons.org/ licenses/by-nc/4.0/) which permits unrestricted noncommercial use, distribution, and reproduction in any medium, provided the original work is properly cited. 
exclusively by culture-positive cases in many developed countries. Second, this study did not compare basic patient characteristics including perinatal factors for the EONS and non-EONS groups. This study also excluded infants with neonatal respiratory diseases such as respiratory distress syndrome, transient tachypnea of the newborn, and congenital pneumonia and included only a small number of preterm infants in the study population.

In conclusion, further studies of the clinical utility of eosinopenia and NLCR as early biomarkers of EONS should focus on invasive bacterial infections leading to critical status and developing therapeutic decision-making strategies.

\section{Conflicts of interest}

No potential conflict of interest relevant to this article was reported.

\section{References}

1. Shane AL, Sánchez PJ, Stoll BJ. Neonatal sepsis. Lancet 2017;390: 1770-80.

2. Drageset M, Fjalstad JW, Mortensen S, Klingenberg C. Management of early-onset neonatal sepsis differs in the north and south of
Scandinavia. Acta Paediatr 2017;106:375-81.

3. Fjalstad JW, Stensvold HJ, Bergseng H, Simonsen GS, Salvesen B, Rønnestad AE, et al. Early-onset sepsis and antibiotic exposure in term infants: a nationwide population-based study in Norway. Pediatr Infect Dis J 2016;35:1-6.

4. Chiesa C, Signore F, Assumma M, Buffone E, Tramontozzi P, Osborn $\mathrm{JF}$, et al. Serial measurements of C-reactive protein and interleukin- 6 in the immediate postnatal period: reference intervals and analysis of maternal and perinatal confounders. Clin Chem 2001;47:1016-22.

5. Robinson P, De SK. How to use... procalcitonin. Arch Dis Child Educ Pract Ed 2018;103:257-62.

6. Shaaban H, Daniel S, Sison R, Slim J, Perez G. Eosinopenia: Is it a good marker of sepsis in comparison to procalcitonin and C-reactive protein levels for patients admitted to a critical care unit in an urban hospital? J Crit Care 2010;25:570-5.

7. Wibrow BA, Ho KM, Flexman JP, Keil AD, Kohrs DL. Eosinopenia as a diagnostic marker of bloodstream infection in hospitalised paediatric and adult patients: a case-control study. Anaesth Intensive Care 2011;39:224-30.

8. Dursun A, Ozsoylu S, Akyildiz BN. Neutrophil-to-lymphocyte ratio and mean platelet volume can be useful markers to predict sepsis in children. Pak J Med Sci 2018;34:918-22.

9. Westerdijk K, Simons KS, Zegers M, Wever PC, Pickkers P, de Jager $\mathrm{CPC}$. The value of the neutrophil-lymphocyte count ratio in the diagnosis of sepsis in patients admitted to the Intensive Care Unit: A retrospective cohort study. PLoS One 2019;14:e0212861.

10. Wilar R. Diagnostic value of eosinopenia and neutrophil to lymphocyte ratio on early onset neonatal sepsis. Korean J Pediatr 2019;62: 217-23. 